\title{
Role of Osteopontin in the Carcinogenesis and Metastasis of Colorectal Cancer
}

\author{
Renhong Huang1, Yingjun Quan' ${ }^{1}$ Ao Huang², Zhijun Min ${ }^{1 *}$ \\ ${ }^{1}$ Department of Gastrointestinal Surgery, Shanghai Pudong Hospital, Fudan University Pudong Medical Center, Shanghai, China \\ ${ }^{2}$ Department of General Surgery, Zhongshan Hospital, Fudan University, Shanghai, China \\ Email:*minzhijun@126.com
}

How to cite this paper: Huang, R.H., Quan, Y.J., Huang, A. and Min, Z.J. (2016) Role of Osteopontin in the Carcinogenesis and Metastasis of Colorectal Cancer. Journal of Cancer Therapy, 7, 729-740. http://dx.doi.org/10.4236/jct.2016.710074

Received: August 29, 2016

Accepted: September 26, 2016

Published: September 29, 2016

Copyright $\odot 2016$ by authors and Scientific Research Publishing Inc. This work is licensed under the Creative Commons Attribution International License (CC BY 4.0).

http://creativecommons.org/licenses/by/4.0/ c) (i) Open Access

\begin{abstract}
Colorectal cancer (CRC) is one of the most malignant tumors in both sexes among all types of tumors worldwide. Up to now, no precise biomarker has been capable of accurately predicting the prognosis for patients with CRC. Therefore, it is essential for us to find a new biomarker to predict the prognosis, overall survival, and disease-free survival of patients with CRC, especially for those patients who have a distant metastasis. There is a growing concern over the issues that osteopontin (OPN), a multifunction phosphorylated protein, has been identified in recent years among multiple tumors types, including CRC. There is increasing evidence showing that high expression of OPN is related to the proliferation, invasion and metastasis of CRC. OPN could support malignant formation by inducing tumor angiogenesis; aggravating the degradation of extracellular matrix; promoting chemo taxis, adhesion, and metastasis of tumors and affecting tumor immunity and apoptosis based on various complex mechanisms. More intensive studies are required in the future to clarify the roles of OPN and its related genes, relevant receptors, protein structure, biological features, and complex molecular mechanisms in colorectal carcinogenesis, as well as to develop beneficial agents for the clinical treatment of CRC to improve patient prognosis.
\end{abstract}

\section{Keywords}

Colorectal Cancer, Osteopontin, Metastasis, Carcinogenesis

\section{Introduction}

Colorectal cancer, one of the most common gastrointestinal neoplasms, remains a leading cause of mortality among all types of tumors according to data from the analysis of patients with CRC in America from 2008 to 2015 despite recent progress in diag- 
nosis and treatment [1]. The survival rate of patients with CRC remains poor as a result of distant metastasis in most cases. Obtaining an early diagnosis and engaging in active clinical treatment measures during the early stages on the basis of tumor staging from the Union for International Cancer Control (UICC) will definitely improve the prognosis, overall survival, and disease-free survival of patients with CRC [2]. The most common histological subtype accounting for approximately $90 \%$ to $95 \%$ of CRCs is adenocarcinoma, including papillary adenocarcinoma, tubular adenocarcinoma, mucinous adenocarcinoma, and signet-ring cell carcinoma. Some common symptoms such as hematochezia, and abdominal discomfort and tests such as digital rectal examination, and abdominal palpation will help to diagnose patients with CRC in the early stage. The exact etiology contributing to CRC is still unclear due to various complex and multi-step mechanisms of CRC development. It is known that many genes including APC, DCC, p53, Ras, and nm23, microstructures and the microenvironment are involved in tumor formation, proliferation, invasion and metastasis [3]-[8], with other factors, such as sex, age, environment, also exerting an influence. The possible treatment options can be used for CRC which are surgical procedures in combination with multi-agent chemotherapeutic medications such as fluorouracil, oxaliplatin, irinotecan, and cetuximab. These medications can be used to either shrink the tumor diameter pre-operation or serve as a post-surgical adjuvant for the treatment of patients with CRC. Colorectal cancer, one of several aggressive tumor types has the propensity to metastasize to the liver, bone, lung, and brain. It appears that local relapse and distant metastasis are intractable problems for treating CRC despite the availability of many new chemotherapeutic medications. Although many parameters such as CEA, CA199, and CA125 are widely used in clinical practice for detection of CRC and patient follow-up, the precise effect and exact clinical value of these parameters are deliberate [9]. Regarding the implementation of an effective treatment in patients with CRC, it is important to elucidate the complex regulatory mechanisms and to identify a novel bio-marker target that can be detected in tumor tissues to locate early tumor cells. Furthermore, finding new markers to predict the prognosis for patients with CRC is also of great importance.

OPN, a phosphorylated protein with multiple functions, has been widely investigated in recent years. It was Sanger who discovered and isolated OPN through the malignant transformation of an epithelial cell line in 1979 [10]. OPN is a member of the small integrin-binding ligand N-liked glycoprotein (SIBLING) family, and is encoded by the OPN gene (spp1) which is mapped to the $\mathrm{q}$ arm of the chromosome 4 and contains seven exons and six introns in the human ortholog. OPN can bind to two receptor families: integrin receptors and CD44 receptors. Integrin receptors comprise an $\alpha$-subunit and $\beta$-subunit, which can give rise tonumerous subtypes (e.g., $\alpha v \beta 1, \alpha v \beta 3, \alpha v \beta 5, \alpha v \beta 6$, $\alpha 8 \beta 1$, and $\alpha 5 \beta 1$ ) that bind with RGD (Arginine-Glycine-Aspartate) sequences in on proteins. In addition, there are other integrin subtypes (e.g., $\alpha 4 \beta 1, \alpha 9 \beta 1$, and $\alpha 4 \beta 7$ ) that can bind to the SVVYGL (Serine-Valine-Valine-Tyrosine-Glutamic-Leucine-Arginine) sequences on OPN [11]. It is known that OPN contains bioactive regions on its surface 
along with its RGD sequences that can exert multiple biological functions such as activation regulatory and structural proteins upon binding with its target receptors, including $\alpha 4 \beta 1, \alpha 9 \beta 1, \alpha v \beta 1, \alpha v \beta 3, \alpha v \beta 5, \alpha v \beta 6, \alpha 8 \beta 1, \alpha 5 \beta 1$, and CD44. These integrin receptors can also in turn active multiple signaling pathways [12] [13]. The N-terminal domain, C-terminal domain and 50 amino acid coding region within the RGD sequences of OPN are highly conserved. The RS locus of the RGD can be disintegrated by thrombin and subsequently form two different sequences, including an N-terminal domain that can bind with RGD sequences, and a C-terminal domain fragment without RGD. OPN, when bound with its specific receptors on cell surface can cause a series of changes such as triggering multiple signal transduction pathways, gene expression and modifications, thus changing the activity of tumor cells [14] [15]. Previous research has shown that OPN can suppress C/EBPs signaling pathways to regulate the multidirectional differentiation capacity of mesenchymal stem cells by binding to integrin $\alpha v \beta 1$ [16]. It is known that $\alpha v \beta 3$ is a vital receptor that can promote tumor metastasis. Studies focused on tumors such as hepatocellular carcinoma demonstrated that the levels of OPN and $\alpha \mathrm{v} \beta 3$ can predict the severity as well as prognosis of patients with hepatocellular carcinoma [17]. Mittlebronn M [18] found that $\alpha \mathrm{v} \beta 3$ and $\alpha v \beta 5$ are expressed in vessels in primary and secondary neoplasms, but the expression of $\alpha v \beta 3$ was more frequent and more pronounced than $\alpha \mathrm{v} \beta 5$ if tumor cells metastasized to central nervous system (CNS). In addition, some other integrin receptors including $\alpha \mathrm{v} \beta 6, \alpha 8 \beta 1$ and $\alpha 5 \beta 1$ can also exert corresponding effects when bound to OPN. Recent studies show that the SVVYGL sequences of OPN may induce angiogenesis and expression of smooth muscle actin (SMA) by bridging the signaling of appropriate receptors, which may provide new insights for the treatment of dilated cardiomyopathy (DCM) disease [19]. CD44 receptors are sulfated adhesion glycoproteins that can participate in the interactions, adhesion and migration of cells. CD44 receptors can regulate intercellular adhesion and signal transduction by activating phosphoinositide 3-kinase/protein kinase $\mathrm{B}(\mathrm{PI} 3 \mathrm{~K} / \mathrm{PKB})$ pathways when bound to OPN. Previous studies indicated that OPN relies on CD44 receptors to active mammalian fibroblasts cell [20]. CD44 receptors are also expressed in acinic cell adenocarcinoma of salivary gland origin and mucoepidermoid carcinoma, as reported in the literature [21]. OPN, a matrix protein with complex structures and strong secretory functions, is expressed in many tissues including mineral compound tissues, epithelial cells, metabolic tissues like intestine, and multiple malignant tumors [22]. OPN is also expressed in macrophages, smooth muscle cells (SMC), T cells and fibroblasts, but the expression levels of OPN is comparatively week under normal circumstances. However, OPN is over expressed under the following conditions: high blood pressure, high blood glucose, oxygen deprivation and in the presence of other microenviroment molecules such as IFN- $\gamma$, FGF-1, ET-1, IGF [23] [24].

Previous studies have demonstrated that OPN is correlated with the formation, proliferation, and invasion of multiple malignant neoplasms such as lung cancer, hepatic carcinoma, gastric cancer, and colorectal cancer, which could represent as an active 
marker for the outcomes of cancer patients [25] [26]. OPN is also related to the tumor stage, tumor grade, lymphatic metastasis and distant metastasis [27]. Therefore, in this view, we mainly discuss the association of OPN with colorectal cancer and the prognostic value, clinical therapeutic function for patients with CRC.

\section{OPN and Carcinogenesis}

Previous studies indicated that the levels of fundamental gene and subsequent protein expression of OPN in tumors and adjacent tissues are relatively higher than in normal tissues. This was observed in lung cancer, gastrointestinal cancer, hepatic cancer, breast cancer, and ovarian cancer [28]-[31]. The formation and progression of tumors require multiple factors and mechanisms, in which OPN can exactly participate in entire process of the pathological mechanisms of tumorigenesis, while yielding complex regulatory effects. OPN is a tumor-related protein that plays a significant role in the adhesion and metastasis of multiple tumors, by regulating tumor adhesion and migration and stimulating tumor angiogenesis.

Tumor angiogenesis is the process in which tumor cells induce the growth of microvessels and to establish localized blood circulation. Nutrition is needed to promote the proliferation, invasion and metastasis of tumor cells. It was demonstrated that VEGF (vascular endothelial growth factor) is a specific HB-EGF (heparin binding epidermal growth factor) that can induce angiogenesis. In addition, VEGF is also a signaling protein that may activate phosphatidylinositol 3 kinase/serine threonine kinase (PI3K/ AKT) pathways, which can promote the expression of VEGF and extracellular signal-regulated kinases (ERK1/2). Previous studies have shown that OPN can stimulate the expression of VEGF, to accelerate tumor angiogenesis by a series of cascade amplification signals. In a research study related to breast cancer [32], OPN can trigger cascading amplification signals by activating the breast tumor kinase/nuclear factor inducing kinase/nuclear factor-kappa B/activating transcription factor-4 signal pathways. OPN plays an important role in the study of articular cartilage, and it was found that OPN induces VEGF expression in articular cartilage through the PI3K/AKT and ERK1/2 signaling pathways [33]. The proliferation, invasion and metastasis of tumor cells are correlated with the process of angiogenesis which is regulated by VEGF. Because OPN induces the expression of VEGF, OPN may become a target for the treatment of various cancers [34]. The extracellular matrix (ECM) is a macromolecular composed by fibronectin, collagens and proteoglycans/glycosaminoglycans, which are synthesized in eukaryotic cells and then secreted to plasma membrane and exposed to either the extra-cellular or intra-cellular environment. The ECM has multiple physiological and cellular influences on growth, differentiation and migration which are important for an organism [35]. It has been demonstrated that extracellular matrix degradation plays an important role in tumor morphology, metastasis and invasion [36]. OPN can function as an extracellular matrix molecule involved in multiple processes such as adhesion and tumor metastasis [37]. Cho A et al. [38] demonstrated that the ECM is an integral component of the tumor environment and is correlated with ova- 
rian cancer progression when it is degraded or eradicated. Cancer requires multiple factors that are capable of degrading the extracellular matrix, and a recent study proved that the metastasis of colorectal cancer is connected with the function of proteolytic enzymes that can degrade the ECM [39]. The chemotaxis, adhesion and metastasis of tumors due to various elements (including growth factors, the extracellular matrix structure and chemokine) [40] [41]. OPN can be an important chemotactic cytokine that is associated with the invasion and metastasis of diverse cancers. Previous studies showed that OPN can trigger signaling cascades that can regulate the chemotaxis, adhesion and metastasis of tumors when bound to its integrin and CD44 receptors. One reported in the recent literature, showed that small integrin binding ligand $\mathrm{N}$-linked glycoproteins (SIBLINGs) which comprise five members (including OPN) are associated with the adhesion, and metastasis of pancreatic cancer [42]. It is widely known that the immune system is a barrier to prevent tumor cell invasion, proliferation and migration. There are many mechanisms that tumors use to escape from immune surveillance within the organism. Previous studies have proven that OPN can increase nitric oxide levels and accelerate tumor apoptosis by up-regulating the expression of nitric oxide syntheses (NOS) and cytochrome c oxide syntheses. OPN is a multifunctional molecule that participate in pathological processes, including immunity, and the metastasis of various tumors. Nagoshi S [43] proved that OPN linked with hepatic inflammation and fibrosis, which may remind us that OPN is presumed to be a target for treating hepatic diseases. Apoptosis is an autonomous, programmed, death process that aids in maintaining homeostasis in an organism. One mechanism by which tumor cells can proliferate, invade and metastasize the inhibition of apoptosis. It was demonstrated that OPN may inhibit tumor apoptosis by increasing $\alpha \mathrm{v} \beta 3$ integrin-mediated Janus kinase 2 (JAK2) phosphorylation and STAT3 activation, whereas another study also showed that OPN knockdown can promote apoptosis of breast cancer cells, while suppressing $\alpha \mathrm{v} \beta 3$ integrin-induced cell invasion and metastasis [11] [44].

\section{Expression of OPN in Colorectal Cancer}

OPN is a significant factor and an important biomarker that may play a vital role in the process of colorectal cancer. Summaries of related foreign and domestic studies from previous years, found that the expression of OPN is upregulated in tumor cells and tissues compared with non-neoplastic tissues.

Likui W et al. [45] found that upregulation of osteopontin mRNA with regard to transcriptional and translation has a significant role in human colorectal cancer, and the expression of OPN is correlated with lymphatic, venous, and lymph node metastasis. Furthermore, patients with high expression of OPN had shorter overall survival rates and disease-free rates than those who had lower expression levels of OPN. Martinez C et al. [46] found that OPN is over expressed in 59 murine intestinal adenomas and that knockdown of OPN could inhibit the proliferation of CRC-based CMT93 cells. Furthermore, OPN may promote the proliferation of murine intestinal tumors via aberrant c-myc signaling, which indicates to us that OPN may serves as a therapeutic tar- 
get for patients with CRC. Imano M et al. [47] found that the presence of OPN in the central tumor area may increase the density of the microvasculature based on the analysis results from 30 CRC patients with liver metastasis and 24 CRC patients without liver metastasis. Previous studies indicated that decreased levels of OPN by RNAi-targeting are associated with the metastatic process of CRC and can inhibit invasion and proliferation as well as enhance the radiosensitivity of human CRC cells [46]. Huang J et al. [48] demonstrated that OPN mRNA and protein were more highly expressed in patients with CRC compared to non-tumor patients, and OPN's target receptors, including integrins and CD44v6, were also highly expressed in metastatic hepatic lesions from patients with CRC. Moreover, OPN can enhance the hepatic metastasis of CRC cells. Fan Y et al. [49] reported that the polymorphisms of OPN are associated with the susceptibility of multiple tumor types, including colorectal cancer, and that the rs 9138 and rs1126616 polymorphisms of OPN may enhance the susceptibility and risk of CRC. $\mathrm{Ng} \mathrm{L}$ et al. [50] found higher post-operative levels of plasma osteopontin (which plays a significant role of CRC metastasis and development) based on statistical data from 79 CRC patients.

Therefore, the higher levels of OPN expression may be correlated with the progression, invasion, proliferation and metastasis of CRC, which remind us that OPN can sever as an important biomarker for the diagnosis and prognosis of patients with CRC.

\section{Role of OPN in Colorectal Cancer and Metastasis}

It is thought that the levels of OPN expression may be related to the clinical pathogenesis and distant metastasis of CRC, but the exact mechanisms still remains controversial. It has been demonstrated that the stem-like nature of colorectal cancer cells are correlated with tumor metastasis and poor survival rate [51].

The stem-like nature of CRC cells has been suggested to correlate with tumor progression, metastasis, and poor survival. Recently, OPN was reported to be related to the stem-like properties in colorectal cancer. The expression of OCT4 and SOX2 were higher in DLD1-cells stably expressing OPN; moreover, the induction effect of OPN on SOX2 was higher than on OCT4, which suggested that cells with high expression levels of OPN possess stem-like properties [51]. During cancer development, cancer stem cells can drive tumor formation as well as metastasis. OPN, and the cytokines hepatocyte growth factor (HGF) and stromal-derived factor 1a (SDF-1), which are secreted from CRC cells, can increase the level of CD44v6 expression in CRC stem cells by activating the $\mathrm{Wnt} / \beta$-catenin pathway, resulting in the metastasis and progression of CRC [22].

In recent years, the incidence and mortality rates for CRC have increased rapidly in Asian countries though there has been a decline on the account of early detection and treatment of CRC [52]. Because the common routes of the metastasis of CRC cells include lymphatic metastasis, hematogenous metastasis and implantation metastasis, the migration of CRC is common in the later stage of the disease and is the primary cause of death in CRC patients. Almost fifty percent of patients had already developed a me- 
tastasis when they were first diagnosed with CRC. Accordingly, patients diagnosed with CRC with hepatic metastasis are often at relatively late stage and have a poor prognosis. It is known that CRC has multiple bio-markers such as carcinoembryonic antigen (CEA) and CA199, which can predict the curative effect as well as the prognosis of patients with CRC either in the late stages or with distant metastasis. However, in the early stages of CRC, these bio-markers can be absent; therefore, it is vital to find a novel molecular marker that could predict and improve the prognosis of patients with CRC. Previous meta-analyses indicated that OPN, a multifunctional phosphorylated protein, could be a new bio-marker to predict the tumor invasion, grade and metastasis as well as patient survival [53]. It was demonstrated that the expression levels of nuclear $\beta$-catenin, CD44 and CD133 were correlated with the progress of liver metastasis [54]. A large proportion of lymphatic metastasis from CRC often emerges in the early stage, which can be one of the most significant prognostic indicators in colorectal cancer [55]. Likui et al. [45] discovered that the expression of OPN mRNA was correlated with lymph node metastasis and that the level of OPN mRNA in patients with CRC was significantly higher than those free of disease, which indicated that OPN plays an important role in predicting CRC.

\section{OPN and Treatment of Colorectal Cancer}

Colorectal cancer is one of the most common malignancies of gastrointestinal cancer, and is a heavy burden to both family and society. Despite the development of modern medical treatment, the overall survival rate remained low, and the therapeutic effects were unsatisfactory due to local relapse, local lymphatic metastasis, distant metastasis to liver, and resistance to chemotherapy and radiotherapy. Detection of the tumor in the early stages may signal a good prognosis for patients with CRC, but the overall prognosis of patients with CRC remains unsatisfactory, the most common treating methods also belongs to surgery and chemotherapy. The early diagnosis and clinical treatment of patients with CRC may have a better survival rate. Li kui et al. indicated that high levels of OPN mRNA expression correlated with the clinicopathological or prognostic significance in patients with CRC [45]. One study found that OPN downregulationOPN by small interfering RNA (siRNA) can cause various effects on the biological properties of CRC cells, including suppression cell growth, invasion, adherence and expression of angiogenic factors [56]. OPN can upregulate the expression of CD44v6 by activating the $\mathrm{Wnt} / \beta$-catenin pathway (which accelerates tumor invasion and metastasis) in colorectal cancer stem cells. Ding L et al. showed that OPN can suppress the gap junction intercellular communication pathway to promote metastasis in the SW480 cell lines, which indicated us that OPN is a potential target for the treatment of patients with CRC [57]. OPN can activate the phosphatidylinositol 3-kinase (PI3K)/Akt pathway to inhibit cancer cell programmed death and promote anchorage-independent growth; evidence shows that inhibition of OPN by siRNA could decrease tumor growth and invasion [58]. A studies of 84 CRC patients showed that OPN was over-expressed in patients who are resistant to oxaliplatin-related chemotherapy treatment [50]. Fan Y et al. 
found that the rs9138 and rs1126616 of OPN were correlated with risk of CRC, and OPN could be a bio-marker of CRC [49].

\section{Conclusions}

Colorectal cancer is one of the most common malignant tumors among various tumors. The incident and mortality of CRC remain high though the development of modern medical technology. Early diagnosis and treatment are essential, and finding a new bio-mark like OPN is also significant for treatment of CRC.

Many studies showed that OPN correlated with the proliferation, invasion and metastasis of multiple tumors including CRC, OPN can be worked as a novel bio-marker in prediction of the prognosis of CRC. CRC patients with lymphatic metastasis have a higher expression of mRNA of OPN than those tissues without lymphatic metastasis, which demonstrated that OPN plays a significant role on the early lymphatic metastasis of CRC. OPN, a multifunction phosphorylated protein, is expected to be a therapy target for the prognosis and treatment of patients with CRC. More researches need to be carried out in order to know about the mechanisms between OPN and CRC, which will bring a better understanding and treatment prospect of CRC.

\section{References}

[1] Ferlay, J., et al. (2015) Cancer Incidence and Mortality Worldwide: Sources, Methods and Major Patterns in GLOBOCAN 2012. International Journal of Cancer, 136, E359-86. http://dx.doi.org/10.1002/ijc.29210

[2] Laubert, T., et al. (2015) Stage-Specific Frequency and Prognostic Significance of Aneuploidy in Patients with Sporadic Colorectal Cancer-A Meta-Analysis and Current Overview. International Journal of Colorectal Disease, 30, 1015-1028. http://dx.doi.org/10.1007/s00384-015-2259-x

[3] King, L.E., et al. (2016) Differential RNA-Seq Analysis Comparing APC-Defective and APC-Restored SW480 Colorectal Cancer Cells. Genomics Data, 7, 293-296. http://dx.doi.org/10.1016/j.gdata.2016.02.001

[4] Jiang, H.W., et al. (2015) Influence of the DCC Gene on Proliferation and Carcinoembryonic Antigen Expression in the Human Colorectal Cancer Cell Line SW1116. Genetics and Molecular Research, 14, 10273-10280. http://dx.doi.org/10.4238/2015.August.28.12

[5] Al-Khayal, K., et al. (2016) Identification of the TP53-Induced Glycolysis and Apoptosis Regulator in Various Stages of Colorectal Cancer Patients. Oncology Reports, 35, 12811286.

[6] Osumi, H., et al. (2016) RAS Mutation Is a Prognostic Biomarker in Colorectal Cancer Patients with Metastasectomy. International Journal of Cancer, 139, 803-811. http://dx.doi.org/10.1002/ijc.30106

[7] Qu, L., et al. (2013) Inhibitory Effect of Upregulated DR-nm23 Expression on Invasion and Metastasis in Colorectal Cancer. European Journal of Cancer Prevention, 22, 512-522. http://dx.doi.org/10.1097/CEJ.0b013e328361625d

[8] Friedman, K., et al. (2016) Medullary Carcinoma of the Colon: A Distinct Morphology Reveals a Distinctive Immunoregulatory Microenvironment. Modern Pathology, 29, 528-541. http://dx.doi.org/10.1038/modpathol.2016.54

[9] Bolocan, A., et al. (2012) Prognostic and Predictive Factors in Colorectal Cancer. Chirurgia 
(Bucur), 107, 555-563.

[10] Senger, D.R., Wirth, D.F. and Hynes, R.O. (1979) Transformed Mammalian Cells Secrete Specific Proteins and Phosphoproteins. Cell, 16, 885-893.

http://dx.doi.org/10.1016/0092-8674(79)90103-X

[11] Zhang, H., et al. (2014) Osteopontin Knockdown Inhibits Alphav, Beta3 Integrin-Induced Cell Migration and Invasion and Promotes Apoptosis of Breast Cancer Cells by Inducing Autophagy and Inactivating the PI3K/Akt/mTOR Pathway. Cellular Physiology and Biochemistry, 33, 991-1002. http://dx.doi.org/10.1159/000358670

[12] Li, Y.S., et al. (2015) Role of Osteopontin in Osteosarcoma. Medical Oncology, 32, 449. http://dx.doi.org/10.1007/s12032-014-0449-y

[13] Sun, C.C., Qu, X.J. and Gao, Z.H. (2016) Arginine-Glycine-Aspartate-Binding Integrins as Therapeutic and Diagnostic Targets. American Journal of Therapeutics, 23, e198-207. http://dx.doi.org/10.1097/MJT.0000000000000053

[14] Beausoleil, M.S., et al. (2011) Deletion of the Thrombin Cleavage Domain of Osteopontin Mediates Breast Cancer Cell Adhesion, Proteolytic Activity, Tumorgenicity, and Metastasis. BMC Cancer, 11, 25. http://dx.doi.org/10.1186/1471-2407-11-25

[15] Stemberger, C., et al. (2014) Osteopontin Is Associated with Decreased Apoptosis and Alphav Integrin Expression in Lung Adenocarcinoma. Acta Histochemica, 116, 222-229. http://dx.doi.org/10.1016/j.acthis.2013.07.009

[16] Chen, Q., et al. (2014) An Osteopontin-Integrin Interaction Plays a Critical Role in Directing Adipogenesis and Osteogenesis by Mesenchymal Stem Cells. Stem Cells, 32, 327-337. http://dx.doi.org/10.1002/stem.1567

[17] Jin, Y., et al. (2014) OPN and $\alpha_{\mathrm{v}} \beta_{3}$ Expression Are Predictors of Disease Severity and Worse Prognosis in Hepatocellular Carcinoma. PLOS ONE, 9, e87930. http://dx.doi.org/10.1371/journal.pone.0087930

[18] Mittelbronn, M., et al. (2013) Expression of Integrins $\alpha_{v} \beta_{3}$ and $\alpha_{v} \beta_{5}$ and Their Ligands in Primary and Secondary Central Nervous System Neoplasms. Histology and Histopathology, 28, 749-758.

[19] Mizuno, Y., et al. (2015) Improvement of Cardiac Function after Implanting the Osteopontin-Derived Peptide SVVYGLR in a Hamster Model of Dilated Cardiomyopathy. Interactive Cardiovascular and Thoracic Surgery, 21, 506-514. http://dx.doi.org/10.1093/icvts/ivv197

[20] Sharon, Y., et al. (2015) Tumor-Derived Osteopontin Reprograms Normal Mammary Fibroblasts to Promote Inflammation and Tumor Growth in Breast Cancer. Cancer Research, 75, 963-973. http://dx.doi.org/10.1158/0008-5472.CAN-14-1990

[21] Fok, T.C., et al. (2014) Expression and Localization of Osteopontin, Homing Cell Adhesion Molecule/CD44, and Integrin $\alpha_{\mathrm{v}} \beta_{3}$ in Mucoepidermoid Carcinoma and Acinic Cell Adenocarcinoma of Salivary Gland Origin. Oral Surgery, Oral Medicine, Oral Pathology and Oral Radiology, 118, 320-329. http://dx.doi.org/10.1016/j.00oo.2014.05.004

[22] Ng, L., et al. (2015) Osteopontin Overexpression Induced Tumor Progression and Chemoresistance to Oxaliplatin through Induction of Stem-Like Properties in Human Colorectal Cancer. Stem Cells International, 2015, Article ID: 247892. http://dx.doi.org/10.1155/2015/247892

[23] Sun, J., et al. (2010) Involvement of Osteopontin Upregulation on Mesangial Cells Growth and Collagen Synthesis Induced by Intermittent High Glucose. Journal of Cellular Biochemistry, 109, 1210-1221. http://dx.doi.org/10.1002/jcb.22503

[24] Oh, K., Seo, M.W., Kim, Y.W. and Lee, D.-S. (2015) Osteopontin Potentiates Pulmonary 
Inflammation and Fibrosis by Modulating IL-17/IFN- $\gamma$-Secreting T-Cell Ratios in Bleomycin-Treated Mice. Immune Network, 15, 142-149.

http://dx.doi.org/10.4110/in.2015.15.3.142

[25] Coppola, D., et al. (2004) Correlation of Osteopontin Protein Expression and Pathological Stage across a Wide Variety of Tumor Histologies. Clinical Cancer Research, 10, 184-190. http://dx.doi.org/10.1158/1078-0432.CCR-1405-2

[26] Fisher, L.W., Jain, A., Tayback, M. and Fedarko, N.S. (2004) Small Integrin Binding Ligand $N$-Linked Glycoprotein Gene Family Expression in Different Cancers. Clinical Cancer Research, 10, 8501-8511. http://dx.doi.org/10.1158/1078-0432.CCR-04-1072

[27] Han, J.C., et al. (2015) Serum Osteopontin Levels Correlate with Clinical and Pathological Features in Non-Small Cell Lung Cancer. Analytical and Quantitative Cytology and Histology, 37, 295-301.

[28] Cho, W.Y., et al. (2015) Suppression of Tumor Growth in lung Cancer Xenograft Model Mice by Poly(Sorbitol-co-PEI)-Mediated Delivery of Osteopontin siRNA. European Journal of Pharmaceutics and Biopharmaceutics, 94, 450-462. http://dx.doi.org/10.1016/j.ejpb.2015.06.017

[29] Lee, S.H., et al. (2015) Ablation of Osteopontin Suppresses $N$-Methyl- $N$-Nitrosourea and Helicobacter Pylori-Induced Gastric Cancer Development in Mice. Carcinogenesis, 36, 1550-1560.

[30] Anborgh, P.H., et al. (2015) Role of Plasma Osteopontin as a Biomarker in Locally Advanced Breast Cancer. American Journal of Translational Research, 7, 723-732.

[31] You, Y., et al. (2015) Higher Matrix Stiffness Upregulates Osteopontin Expression in Hepatocellular Carcinoma Cells Mediated by Integrin Beta1/Gsk3Beta/Beta-Catenin Signaling Pathway. PLoS ONE, 10, e0134243. http://dx.doi.org/10.1371/journal.pone.0134243

[32] Chakraborty, G., Jain, S., and Kundu, G.C. (2008) Osteopontin Promotes Vascular Endothelial Growth Factor-Dependent Breast Tumor Growth and Angiogenesis via Autocrine and Paracrine Mechanisms. Cancer Research, 68, 152-161. http://dx.doi.org/10.1158/0008-5472.CAN-07-2126

[33] Xu, J., et al. (2015) Osteopontin Induces Vascular Endothelial Growth Factor Expression in Articular Cartilage through PI3K/AKT and ERK1/2 Signaling. Molecular Medicine Reports, 12, 4708-4712. http://dx.doi.org/10.3892/mmr.2015.3975

[34] Bandopadhyay, M., et al. (2014) Osteopontin as a Therapeutic Target for Cancer. Expert Opinion on Therapeutic Targets, 18, 883-895. http://dx.doi.org/10.1517/14728222.2014.925447

[35] Theocharis, A.D., Skandalis, S.S., Gialelia, C. and Karamanos, N.K. (2016) Extracellular Matrix Structure. Advanced Drug Delivery Reviews, 97, 4-27. http://dx.doi.org/10.1016/j.addr.2015.11.001

[36] Tzedakis, G., et al. (2015) The Importance of Neighborhood Scheme Selection in AgentBased Tumor Growth Modeling. Cancer Informatics, 14, 67-81.

[37] Li, Y., et al. (2015) Osteopontin Promotes Invasion, Migration and Epithelial-Mesenchymal Transition of Human Endometrial Carcinoma Cell HEC-1A through AKT and ERK1/2 Signaling. Cellular Physiology and Biochemistry, 37, 1503-1512. http://dx.doi.org/10.1159/000438518

[38] Cho, A., Howell, V.M., and Colvin, E.K. (2015) The Extracellular Matrix in Epithelial Ovarian Cancer-A Piece of a Puzzle. Frontiers in Oncology, 5, 245.

http://dx.doi.org/10.3389/fonc.2015.00245

[39] Zhang, Y.Y., Chen, B., and Ding, Y.Q. (2012) Metastasis-Associated Factors Facilitating the 
Progression of Colorectal Cancer. Asian Pacific Journal of Cancer Prevention, 13, 24372344. http://dx.doi.org/10.7314/APJCP.2012.13.6.2437

[40] Guan, X. (2015) Cancer Metastases: Challenges and Opportunities. Acta Pharmaceutica Sinica $B, 5,402-418$. http://dx.doi.org/10.1016/j.apsb.2015.07.005

[41] Shevde, L.A. and Samant, R.S. (2014) Role of Osteopontin in the Pathophysiology of Cancer. Matrix Biology, 37, 131-141. http://dx.doi.org/10.1016/j.matbio.2014.03.001

[42] Kaleagasioglu, F. and Berger, M.R. (2014) SIBLINGs and SPARC Families: Their Emerging Roles in Pancreatic Cancer. World Journal of Gastroenterology, 20, 14747-14759. http://dx.doi.org/10.3748/wjg.v20.i40.14747

[43] Nagoshi, S. (2014) Osteopontin: Versatile Modulator of Liver Diseases. Hepatology Research, 44, 22-30. http://dx.doi.org/10.1111/hepr.12166

[44] Behera, R., et al. (2010) Activation of JAK2/STAT3 Signaling by Osteopontin Promotes Tumor Growth in Human Breast Cancer Cells. Carcinogenesis, 31, 192-200. http://dx.doi.org/10.1093/carcin/bgp289

[45] Wang, L., Wang, H. and Zhang, S. (2010) Clinical Significance of the Upregulated Osteopontin mRNA Expression in Human Colorectal Cancer. Journal of Gastrointestinal Surgery, 14, 74-81. http://dx.doi.org/10.1007/s11605-009-1035-Z

[46] Martinez, C., Churchman, M., Freeman, T. and Ilyas, M. (2010) Osteopontin Provides Early Proliferative Drive and May Be Dependent upon Aberrant C-Myc Signalling in Murine Intestinal Tumours. Experimental and Molecular Pathology, 88, 272-277. http://dx.doi.org/10.1016/j.yexmp.2009.12.008

[47] Imano, M., et al. (2010) Increased Osteopontin-Positive Macrophage Expression in Colorectal Cancer Stroma with Synchronous Liver Metastasis. World Journal of Surgery, 34, 1930-1936. http://dx.doi.org/10.1007/s00268-010-0582-5

[48] Huang, J., Pan, C., Hu, H., Zheng, S. and Ding, L. (2012) Osteopontin-Enhanced Hepatic Metastasis of Colorectal Cancer Cells. PLOS ONE, 7, e47901. http://dx.doi.org/10.1371/journal.pone.0047901

[49] Fan, Y., et al. (2013) The Polymorphisms of Osteopontin Gene and Plasma Osteopontin Protein Levels with Susceptibility to Colorectal Carcinoma. DNA and Cell Biology, 32, 594600. http://dx.doi.org/10.1089/dna.2013.2090

[50] Ng, L., et al. (2015) Post-Operative Plasma Osteopontin Predicts Distant Metastasis in Human Colorectal Cancer. PLOS ONE, 10, e0126219. http://dx.doi.org/10.1371/journal.pone.0126219

[51] Kashihara, H., et al. (2014) CD133 Expression Is Correlated with Poor Prognosis in Colorectal Cancer. Hepato-Gastroenterology, 61, 1563-1567.

[52] Todaro, M., et al. (2014) CD44v6 Is a Marker of Constitutive and Reprogrammed Cancer Stem Cells Driving Colon Cancer Metastasis. Cell Stem Cell, 14, 342-356. http://dx.doi.org/10.1016/j.stem.2014.01.009

[53] Sung, J.J., et al. (2008) Asia Pacific Consensus Recommendations for Colorectal Cancer Screening. Gut, 57, 1166-1176. http://dx.doi.org/10.1136/gut.2007.146316

[54] Zhao, M., Liang, F., Zhang, B., Yan, W. and Zhang, J. (2015) The Impact of Osteopontin on Prognosis and Clinicopathology of Colorectal Cancer Patients: A Systematic Meta-Analysis. Scientific Reports, 5, Article Number: 12713. http://dx.doi.org/10.1038/srep12713

[55] Michl, M., et al. (2015) Expression of Cancer Stem Cell Markers in Metastatic Colorectal Cancer Correlates with Liver Metastasis, but Not with Metastasis to the Central Nervous System. Pathology Research and Practice, 211, 601-609.

http://dx.doi.org/10.1016/j.prp.2015.05.006 
[56] Wu, X.L., et al. (2014) Osteopontin Knockdown Suppresses the Growth and Angiogenesis of Colon Cancer Cells. World Journal of Gastroenterology, 20, 10440-10448. http://dx.doi.org/10.3748/wjg.v20.i30.10440

[57] Ding, L., Hu, H.G., and Zheng, S. (2011) Effects of Osteopontin on Gap Junctional Intercellular Communication in Colon Cancer Cell Lines. National Medical Journal of China, 91, 2578-2581.

[58] Shao, J., Kay Washington, M., Saxena, R. and Sheng, H. (2007) Heterozygous Disruption of the PTEN Promotes Intestinal Neoplasia in $A P C^{\mathrm{min} /+}$ Mouse: Roles of Osteopontin. Carcinogenesis, 28, 2476-2483. http://dx.doi.org/10.1093/carcin/bgm186

\section{Submit or recommend next manuscript to SCIRP and we will provide best service} for you:

Accepting pre-submission inquiries through Email, Facebook, LinkedIn, Twitter, etc. A wide selection of journals (inclusive of 9 subjects, more than 200 journals)

Providing 24-hour high-quality service

User-friendly online submission system

Fair and swift peer-review system

Efficient typesetting and proofreading procedure

Display of the result of downloads and visits, as well as the number of cited articles Maximum dissemination of your research work

Submit your manuscript at: http://papersubmission.scirp.org/

Orcontact jct@scirp.org 